\title{
TCS-CAIN: NIR survey of the Galactic plane
}

\author{
C. González-Fernández ${ }^{1}$, A. Cabrera-Lavers ${ }^{1,2}$, F. Garzón ${ }^{1,3}$, \\ P. L. Hammersley ${ }^{1}$, M. López-Corredoira ${ }^{1}$ and B. Vicente ${ }^{1}$ \\ ${ }^{1}$ Instituto de Astrofísica de Canarias, E-38205 La Laguna, Tenerife, Spain \\ ${ }^{2}$ GTC Project Office, E-38205 La Laguna, Tenerife, Spain \\ ${ }^{3}$ Departamento de Astrofísica, Universidad de La Laguna, E-38205 La Laguna, Tenerife, Spain

\begin{abstract}
We present TCS-CAIN, a NIR survey of the Galactic plane, recently made public at the Instituto de Astrofísica de Canarias, and some results derived from it: with star counts derived directly the structure of the inner the Milky Way can be dissected, obtaining also estimations for the extinction toward the inner MW.
\end{abstract}

Keywords. Surveys, Galaxy: structure, Galaxy: bulge, Galaxy: stellar content, Stars: distances

\section{Introduction: the catalogue}

TCS-CAIN is a deep multi-color NIR survey. Mapping selected areas along the Galactic plane, it reaches deeper than 2MASS or DENIS. The main scientific goal of TCS-CAIN is to study the structure of the Milky Way, mainly the bulge/bar and the disk, so the fields to observe have been chosen to sample well enough the $0^{\circ}<\mathrm{l}<35^{\circ}$ zone and to give enough coverage both in 1 and $b$ of the disk.

The body of the catalogue comprises about 500 fields, $4.25^{\prime} \times 4.25^{\prime}$ each, obtained using JHKs photometry in the TCS Telescope (1.5m, Tenerife, Spain) yielding 10 million source points, with limiting magnitudes of $17(\mathrm{~J}), 16.5(\mathrm{H})$ and $15.2(\mathrm{Ks})$, and with a photometric precision of $0.1 \mathrm{mag}$ (against $2 \mathrm{MASS}$ data). The spatial resolution is $1^{\prime}$, with astrometric errors of $0.15^{\prime}$ for the position of the point sources.

\section{Results: the bar}

Hammersley et al. (2000), studying red giant star counts, spotted the presence of an overdensity from $l=27^{\circ}$ to $l=5^{\circ}$ at different magnitudes. Assuming an extinction law, the magnitude of this overdensity can be translated into distances along different lines of sight, rendering a elongated feature that runs from $l=5^{\circ}$ to $l=27^{\circ}$ with a position angle of $43^{\circ} \pm 7^{\circ}$ and a half-length of $4 \mathrm{kpc}$, that may be interpreted as a bar.

This structure can be traced further making use of the red clump stars. Since their luminosity function is rather narrow, they present a well-defined clump in the colormagnitude diagrams (CMD). It is possible then to extract star density and interstellar extinction by isolating this clump on a CMD (López-Corredoira et al. (2002) for details).

We can obtain a magnitude estimate for the red clump stars, which can be translated into a distance with the estimate of the extinction. Since TCS-CAIN has a good coverage of the inner parts of the Milky Way, we can apply this analysis to several fields in various lines of sight, and compare them with similar studies, as can be seen in Fig. 1, left panel. Clear traces of a long structure appear along the plane, reaching almost $1=30^{\circ}$, and with an angle of $43^{\circ} \pm 3^{\circ}$, in concordance with that proposed by Hammersley et al. (2000).

\section{Extinction towards the inner Milky Way}

With TCS-CAIN DCM's it is possible to obtain an extinction estimate along the desired line of sight, without any prior assumption of the stellar or interstellar matter 

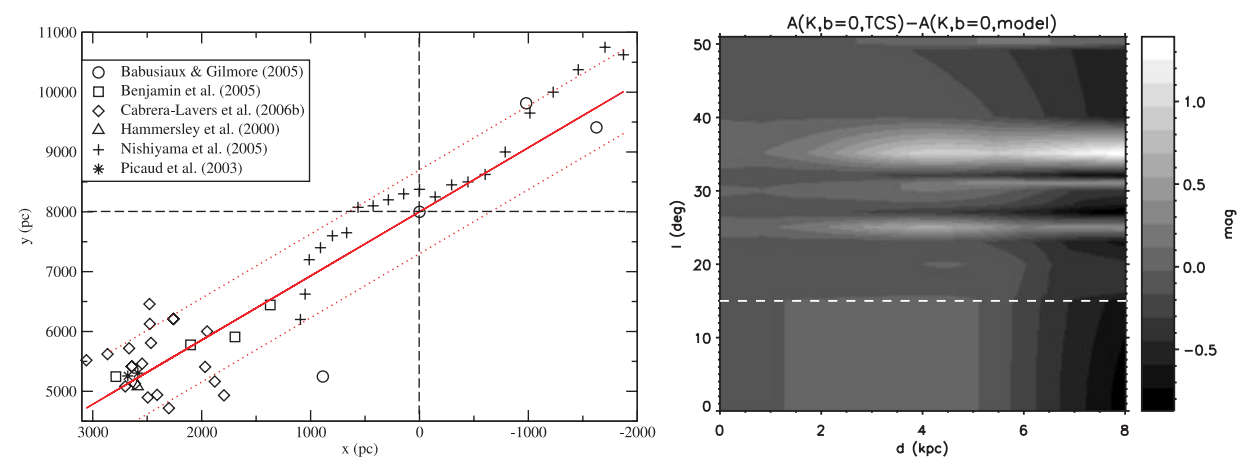

Figure 1. Left: Distance estimations for the red clump giants. Data are taken from Nishiyama et al. (2005), Babusiaux \& Gilmore (2005), Benjamin et al. (2005), Picaud et al. (2003), López-Corredoira et al. (2007) and Hammersley et al. (2000). A feature with an angle of $43^{\circ}$ and a width of $1 \mathrm{kpc}$ is marked. Right: The difference in magnitude between the modelled values in Deguchi et al. (2004) and the ones derived with TCS-CAIN. The white line denotes the lower Galactic longitude limit of our present calculations.

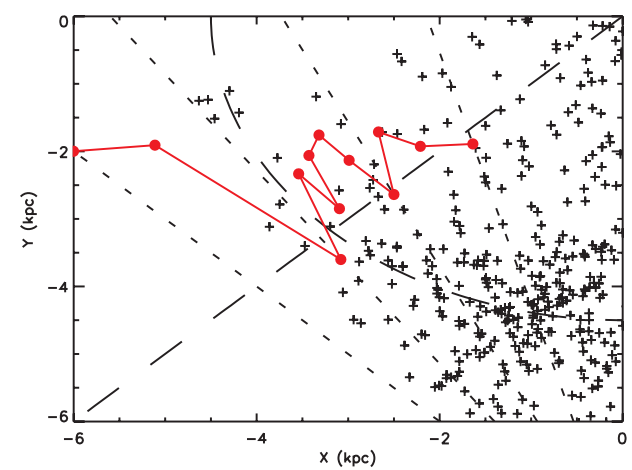

Figure 2. New distance estimation for the Deguchi et al. (2004) data, based in our derived extinctions. Red line and dots denote the completeness limit of our calculations.

distribution and thus, among other things, reproduce the patchy nature of the dust distribution at certain Galactic longitudes. Differences between models and our direct measurements can add up to $2 \mathrm{mag}$ in $\mathrm{A}_{K}$. With this extinction, it is possible to derive a new distance to the Deguchi et al. (2004) masers (see Fig. 2).

\section{References}

Babusiaux, C., \& Gilmore, G. 2005, MNRAS, 358, 1309

Benjamin, R. A., et al. 2005, ApJ, 630, L149

Cabrera-Lavers, A., Garzón, F., Hammersley, P. L., Vicente, B., \& González-Fernández, C. 2006, $A \mathscr{E} A, 453,371$

Deguchi, S., et al. 2004, PASJ, 56, 765

Hammersley, P. L., Garzón, F., Mahoney, T. J., López-Corredoira, M., \& Torres, M. A. P. 2000, MNRAS, 317, L45

López-Corredoira, M., Cabrera-Lavers, A., Garzón, F., \& Hammersley, P. L. 2002, A\&\&A, 394, 883

López-Corredoira, M., Cabrera-Lavers, A., Mahoney, T. J., Hammersley, P. L., Garzón, F., \& González-Fernández, C. 2007, AJ, 133, 154

Nishiyama, S., et al. 2005, ApJ, 621, L105

Picaud, S., Cabrera-Lavers, A., \& Garzón, F. 2003, A\&A, 408, 141 